\title{
O Problema do Jogo do Balanceamento de Carga
}

\author{
Eric Batista da Silva ${ }^{1}$, Daniel Cordeiro ${ }^{1}$ \\ ${ }^{1}$ Escola de Artes, Ciências e Humanidades - Universidade de São Paulo (EACH-USP) \\ \{ericbatista.silva, daniel.cordeiro\}@usp.br
}

\begin{abstract}
This work presents the undergraduate research project about the Selfish Load Balancing problem, a game modeled with Algorithmic Game Theory that has applications on High Performance Computing. This paper presents the problem and a preliminary experimental evaluation of its use in practice.

Resumo. Neste trabalho de Iniciação Científica estudamos o Jogo de Balanceamento de Carga, um jogo modelado com Teoria Algorítmica dos Jogos que possui aplicações práticas em Computação de Alto Desempenho. Este texto apresenta sua modelagem teórica, bem como a avaliação experimental preliminar de seu uso na prática.
\end{abstract}

\section{Introdução}

O problema do balanceamento de carga é fundamental em sistemas distribuídos. O balanceamento de carga consiste em distribuir um conjunto de tarefas com custos associados (chamado também de pesos), em um conjunto de processadores de forma que a carga de trabalho seja equilibrada entre os processadores. Este é um problema NP-difícil clássico de Teoria do Escalonamento que, neste trabalho, abordaremos sob a ótica de Teoria Algorítmica dos Jogos.

Com o crescimento rápido e descentralizado da rede, surgiram diversos problemas computacionais nos quais não há um controle centralizado dos agentes envolvidos, sendo que, vários desses agentes tem interesses próprios e suas ações impactam os demais. Tais problemas motivaram a criação da Teoria Algorítmica dos Jogos [Nisam et al. 2007], uma combinação de duas grandes áreas: Teoria dos Jogos e Computação. Com o uso de Teoria dos Jogos como ferramenta para estudar as relações de tais agentes, foi possível aplicá-las no estudo desses problemas de computação.

O objetivo deste trabalho é estudar o problema clássico de Teoria Algorítmica dos Jogos conhecido como o Jogo de Balanceamento de Carga. Para tanto, é necessário estudar os conceitos relacionados à Teoria dos Jogos, uma área da matemática aplicada, e sua extensão para a Computação, chamada de Teoria Algorítmica dos Jogos. Tais conceitos nos permitem modelar agentes inteligentes e egoístas, que podem tomar decisões de forma totalmente decentralizada. Neste projeto de Iniciação Científica, estudamos como o problema de Balanceamento de Carga pode ser modelado como um jogo onde cada processo é associado a um agente que definirá em qual máquina a tarefa deverá ser executada. Além disso, apresentamos uma análise experimental preliminar do uso de tal jogo em workloads simples para compreender melhor a aplicabilidade da técnica na prática.

\section{Teoria dos Jogos}

Teoria dos Jogos é uma área estudada na matemática aplicada que tem como objetivo estudar fenômenos resultantes da interação entre indivíduos livres, capazes de realizar 
escolhas com o intuito de atingir um objetivo específico. Quando aplicada em conjunto com a área da Computação, é conhecida como Teoria Algorítmica dos Jogos. Em nosso trabalho, usaremos ela para modelar o problema de escalonamento conhecido como o problema do Balanceamento de Carga, um problema clássico, e ainda assim recorrente, de plataformas de computação de alto desempenho.

Os indivíduos livres estudados são conhecidos como jogadores em Teoria dos Jogos. Assume-se que estes são indivíduos racionais, com autonomia para realizar escolhas e que estão interessados em atingir um objetivo bem definido. Além disso, considera-se que esses indivíduos realizam suas escolhas com o intuito de satisfazer seus objetivos de maneira egoísta, considerando o conhecimento deles ou as expectativas a respeito das escolhas dos demais jogadores.

Um jogo é o conjunto de regras que define a interação estratégica entre os jogadores. Ele descreve os limites das escolhas dos jogadores, além dos objetivos de cada um, sem especificar quais escolhas cada jogador deve fazer para atingir seus objetivos. Teoria dos Jogos estuda os resultados que são obtidos a partir das interações que seguem o conjunto de regras definidos no jogo. É importante ressaltar que cada jogador realiza sua escolha de forma independente, além de que todas as escolhas são feitas simultaneamente.

Formalmente, um jogo $J$ consiste em um conjunto $N=\{1,2, \ldots, n\}$ de jogadores. Cada jogador $i$ possui um conjunto próprio de possíveis estratégias, denominado $S_{i}$. Para jogar o jogo, cada jogador $i$ escolhe uma estratégia $s_{i} \in S_{i}$. Usa-se $s=\left(s_{1}, s_{2}, \ldots, s_{n}\right)$ para denotar um vetor de estratégias, com as estratégias selecionadas por cada jogador e $S=X_{i} S_{i}$ para denotar todas as possíveis combinações nas quais os jogadores podem escolher suas estratégias. Além disso, denotamos $S_{-i}$ como o vetor de dimensão $(n-1)$ de estratégias escolhidas por todos os jogadores exceto o jogador $i$ [Cordeiro 2012].

O vetor de estratégias $s$ pertencente a $S$ selecionado pelos jogadores determina o resultado para cada jogador. Cada jogador escolhe sua estratégia com base em uma lista de preferências ordenada de acordo com o resultado obtido. Uma forma simples de se estabelecer essas preferências é associar um valor para cada resultado. Normalmente, isso é feito a partir de uma função utilitária. [Cordeiro 2012]

É importante ressaltar que a função utilitária de cada jogador depende não somente da estratégia do jogador em questão, mas também de todas as outras estratégias escolhidas por todos os jogadores. Neste trabalho, focamos nos casos em que as estratégias são selecionadas de forma determinística pelos jogadores. Uma decisão determinística feita por um jogador é chamada de estratégia pura [Cordeiro 2012].

Nós dizemos que um jogo possui uma solução de estratégia dominante se cada jogador tem uma única melhor estratégia, independente das estratégias dos outros jogadores. Jogos com uma solução de estratégia dominante são raros. Na maioria dos casos, buscamos por uma solução em Teoria dos Jogos na qual cada indivíduo chega a um consenso, enquanto maximiza sua função utilitária. A ideia é encontrar uma solução na qual nenhum jogador consegue realizar outra escolha de forma a maximizar sua função utilitária. Este conceito central de Teoria dos Jogos é conhecido como Equilíbrio de Nash [Cordeiro 2012].

Um Equilíbrio de Nash ocorre quando nenhum jogador $i$ tem qualquer incentivo 
em mudar sua estratégia de atual, de forma que melhora de forma unilateral sua função utilitária. É fácil perceber que uma solução de estratégia dominante é um Equilíbrio de Nash. Mas, de forma geral, é preciso estudar o jogo para determinar a existência de um Equilíbrio de Nash. Uma instância de um jogo pode ter uma única configuração de Equilíbrio de Nash, múltiplas configurações ou não ter nenhuma.

Em nosso estudo, se faz importante o uso do conceito de Preço da Anarquia. Em suma, trata-se de uma forma de medir o quão ineficiente é a solução obtida por um sistema no qual cada jogador realiza sua escolha de forma egoísta, em comparação com uma solução idealizada.

Definição 2.1. (Preço da Anarquia) Dado um conjunto $\mathrm{S}$ de possíveis estratégias, $E \subseteq S$ o conjunto de todos os equilíbrios de nash e $C: S \mapsto R$ a função de custo que mede a ineficiência do sistema, o preço da anarquia é definido como:

$$
P d A=\frac{\max _{s \in E} C(s)}{\min _{s \in S} C(s)}
$$

\subsection{Jogo de Balanceamento de Carga}

Neste trabalho, abordamos o jogo do balanceamento de carga. O balanceamento de carga é normalmente visto como um problema de algoritmos. Uma instância do problema pode ser descrita por um conjunto de tarefas com pesos diferentes que devem ser distribuídas entre um conjunto de máquinas, possivelmente com velocidades diferentes, de forma que a carga é distribuída igualmente entre as máquinas. Um dos objetivos clássicos desse problema é minimizar a carga máxima entre todas as máquinas, objetivo esse denominado de makespan.

Em Teoria dos Jogos, esse problema é abordado de uma forma diferente. Assumese que cada tarefa é administrada por um jogador, que tem como objetivo fazer com que a tarefa seja executada na máquina com a menor carga possível. Com isso, o Equilíbrio de Nash desse jogo é comparado com a solução ótima que satisfaz a condição de minimizar a carga máxima entre todas as máquinas. A relação entre o pior caso no Equilíbrio de Nash e a solução ótima é chamado de Preço da Anarquia [Nisam et al. 2007]. Neste trabalho assumimos que as máquinas são idênticas e que os jogadores escolhem aleatoriamente uma máquina do conjunto de máquinas com menor carga ao determinar sua estratégia.

\section{Avaliação experimental}

Implementamos um simulador para analisar a qualidade do makespan obtido com a execução do jogo do balanceamento de carga. A Figura 1 mostra o resultado obtido com o jogo ao distribuir-se 10 tarefas de peso 1 em 5 máquinas idênticas.

Este é um exemplo no qual o makespan coincidiu com o melhor caso, ou seja, o preço da anarquia foi igual a 1. Porém, tal situação se faz muito incomum, uma vez que geralmente os processos possuem cargas diferentes.

O resultado é diferente para uma instância com 5 máquinas idênticas, 5 tarefas com peso 1 e mais 5 tarefas com peso 2. A Figura 2 a mostra o resultado ótimo para o problema, enquanto que a Figura $2 \mathrm{~b}$ mostra o resultado obtido pelo jogo. 


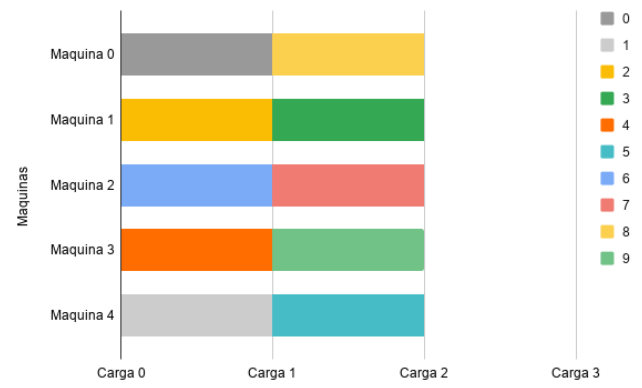

Figura 1. Alocação obtida com tarefas e máquinas idênticas.

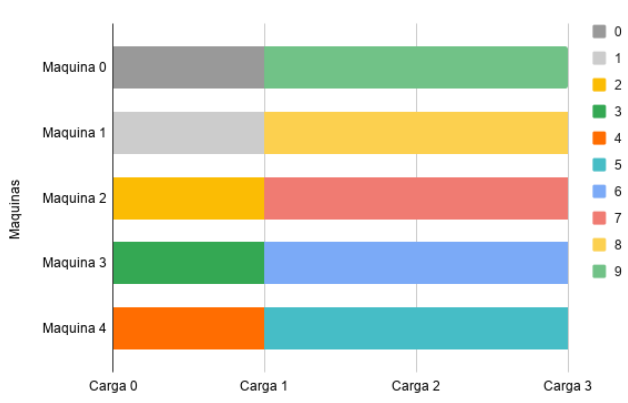

(a) Solução Ótima

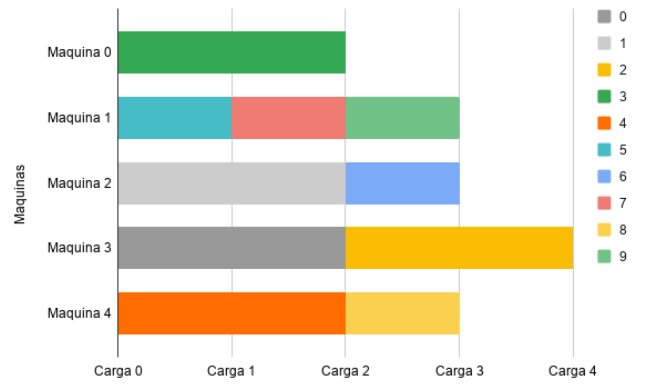

(b) Solução Obtida

Figura 2. Alocação dos processos nas máquinas: ótimo vs. obtido.

O uso do jogo resultou em um makespan $25 \%$ pior do que a melhor solução para o problema. O resultado ilustra um aspecto prático do uso do Jogo do Balanceamento de Carga: na prática, raramente se consegue atingir uma solução ótima rapidamente (já que o problema é NP-difícil); porém, o uso do jogo mostra que é possível conceber um algoritmo distribuído onde a decisão é tomada por cada tarefa de forma independente, mas o resultado final pode não ser ótimo.

\section{Conclusão e trabalhos futuros}

Neste trabalho estudamos a formulação teórica do problema do Jogo do Balanceamento de Carga e analisamos experimentalmente sua aplicação no balanceamento de tarefas. Ainda que o resultado obtido pelo jogo não seja o ótimo, o modelo permite conceber um algoritmo totalmente distribuído onde cada agente (tarefa) decide de forma independente em qual máquina cada tarefa será executada.

Como trabalho futuro pretendemos estudar a aplicação do jogo em traços reais de aglomerados de computação utilizados em plataformas de computação em nuvem.

\section{Referências}

Cordeiro, D. (2012). The impact of cooperation on new high performance computing platforms. PhD thesis, Université de Grenoble.

Nisam, N., Roughgarden, T., Tardos, É., and Vazirani, V. V. (2007). Algorithmic Game Theory. Cambridge University Press, New York, NY, USA. 